De retour au laboratoire, les tampons ont été séchés à l'air et à l'étuve, puis pesés. La différence entre le poids du tampon chargé de cérumen et celui du témoin correspondant constituait la mesure de l'importance de la secrétion auriculaire.

A titre de vérification, les mêmes opérations ont été tentées sur les mêmes vaches, à deux années d'intervalle. Les chiffres obtenus, bien que légèrement différents, se sont montrés-suffisamment rapprochés les uns des autres pour mériter d'être pris en considération.

(A suivre)

\title{
ENQUÊTE SUR LES LAITS VENDUS A LILLE
}

\author{
par Marcel PAGET
}

Maître de Conférences à la Faculté Libre de Médecine et de Pharmacie de Lillé(1).

A l'heure où l'allaitement naturel est de plus en plus délaissé pour l'allaitement artificiel, il m'a paru intéressant d'étudier au point de vue chimique, la composition du lait de vache vendu à Lille, soit par les laitiers ambulants, soit par leurs intermédiaires (les épiciers détaillants).

En vue de cette étude, j'ai donc procédé à l'achat d'échantillons de lait entier (du moins vendu comme tel), prélevés à diverses périodes de l'année dans les différents quartiers de la ville.

Quarante-cinq prélèvements ont été ainsi effectués.

Les recherches analytiques que j'ai poursuivies visèrent surtout la matière grasse, le lactose et les chlorures (la connaissance du taux de ces deux derniers éléments $m$ 'étant, en effet, nécessaire pour l'évaluation de la constante moléculaire simplifiée apparente dont la valeur fournit actuellement la donnée la plus sûre pour l'appréciation du mouillage du'lait.)

A titre documentaire, j'indique les techniques de dosage que j'ai adoptées :

Le dosage de la matière grasse fut opéré à la fois par le procédé Gerber et par une méthode personnelle que j'ai publiée dans l'Union Pharmaceutique d'août 1927 (méthode d'exécution un peu longue, mais d'une précision rigoureuse et qui nécessite le mélange d'un certain volume de lait avec du sulfate de soude anhydre, puis épuisement par l'éther du magma ainsi obtenu. L'opération se faisait dans un lixiviateur Soxhlet).

Le dosage du lactose fut effectué par la méthode de Bertrand, les laits ayant été au préalable déféqués par la méthode Carrez au ferrocyanure de zinc. Quant au taux de chlorures, il fut déterminé tout d'abord par la méthode de Denigès, puis (dans mes dernières analyses) par une technique sûre et rapide que publiera prochainement mon collègue le Professeur Raquet.

(1) Extrait du Journal des Sciences Médicales de Lille, 27 novernbre 1927. 
Les résultats que j’ai obtenus sont condensés dans le tableau suivant, dont la lecture est particulièrement suggestive.

Je n'y ai reproduit que les chiffres sans décimales trouvés pour les taux de matière grasse et la constante moléculaire simplifiée :

\begin{tabular}{|c|c|c|c|c|c|c|c|c|}
\hline Echantillons & M. $\mathrm{G}$. & (c. M. S. & Echantillons & M. G. & C. M. $\mathrm{s}$. & Echantillons & M. G. & c. M. s. \\
\hline No 1 & 27 & 65 & No 16 & 25 & 66 & No 31 & 25 & 70 \\
\hline No 2 & 25 & 63 & No 17 & 30 & 66 & No 32 & 24 & 61 \\
\hline No 3 & 25 & 68 & No 18 & 35 & 71 & No 33 & 25 & 71 \\
\hline No 4 & 45 & 73 & No 19 & 37 & 75 & No 34 & 40 & 76 \\
\hline No 5 & 27 & 70 & No 20 & 23 & 65 & No 35 & 19 & 61 \\
\hline No 6 & 24 & 63 & No 21 & 26 & 69 & No 36 & 27 & 72 \\
\hline No 7 & 31 & 69 & No 22 & 25 & 69 & No 37 & 39 & 63 \\
\hline No 8 & 27 & 61 & No 23 & 31 & 68 & No 38 & 14 & 59 \\
\hline No 9 & 26 & 59 & No 24 & 28 & 70 & No 39 & 24 & .68 \\
\hline No 10 & 23 & 62 & No 25 & 24 & 61 & No 40 & 21 & 69 \\
\hline № 11 & 26 & 69 & Na 26 & 36 & 72 & No 41 & 8 & 53 \\
\hline No 12 & 20 & 58 & No 27 & 37 & 69 & No 42 & 18 & 58 \\
\hline No 13 & 27 & 69 & No 28 & 25 & 67 & No 43 & 24 & 68 \\
\hline No 14 & 25 & 70 & No 29 & 39 & 71 & No 44 & 30 & 69 \\
\hline No 15 & 25 & 70 & No 30 & 17 & 53 & No 45 & 23 & 60 \\
\hline
\end{tabular}

Si l'on effectue le décompte des laits de taux butyreux supérieur à 30 grammes, on arrive à un total réellement impressionnant : douze échantillons présentent une teneur en matière grasse au moins égale à cette moyenne et trente-trois un taux nettement inférieur. Ce qui conduit à évaluẹ à $75 \%$ le pourcentage des laits accusant à l'analyse un taux butyreux laissant présumer une soustraction de crème, c'est-àdire une fraude.

Un pourcentage aussi élevé ne s'explique que par un écrémage exagéré de ces laits. C'est précisément ce que je me propose de démontrer.

Les laits couramment vendus à Lille sont, en effet, des laits de petit ou de moyen mélange (1). Or, si l'on compare leur physionomie-butyreuse (telle qu'elle ressort du tableau précédent) à celle des laits de même catégorie, prélevés directement à l'étable, l'on ne peut manquer d'être surpris par leur dissemblance. Alors que les taux butyreux inférieurs à 30 grammes sont légion chez ceux-là, ils sont au contraire inexistants chez ceux-ci. Les remarquables travaux de Porcher et de beaucoup d'autres auteurs l'attestent bien. J'ai eu d'ailleurs l'occasion de mettre ce fait en évidence au cours de multiples essais que j'ai effectués précédemment et dont l'étude critique a fait l'objet d'un mémoire que j'ai

(1) J'entends par laits de petit mélange les laits de mélange de 10 à 12 vaches - laits de moyen mélange, de 12 à 50 vaches. 
publié en juin dernier (1). A côté d'autres questions élucidées dans ce travail j'ai délimité, en particulier, la marge exacte dans laquelle évoluaient les taux butyreux de ces laits purs. J'y ai démontré : $1^{\circ}$ que les laits de petit mélange (et $a$ fortiori de moyen mélange), provenant des traites du matin, accusaient toujours à l'analyse une teneur en matière grasse au moins égale à 30 grammes (cette moyenne devant être considérée comme un chiffre minimum rarement atteint; $2^{\circ}$ que cette - moyenne inférieure n'était jamais rencontrée dans les laits provenant du mélange intégral de toutes les traites de la journée pour lesquels le taux minimum était voisin de $\mathbf{3 0}$ grammes et le taux moyen compris entre 33 et 36 grammes.

L'ensemble de ces données m'autorise done à conclure que, puisque les laits vendus à Lille entrent dans la catégorie des laits précédents (laits de petit et moyen mélange), le consommateur est en droit d'exiger que leurs caractéristiques analytiques soient normales. Or, l'examen du tableau ci-dessus montre suffisamment que ces exigences très justifiables sont loin d'être satisfaites.

$75 \%$ des laits que j'ai analysés ont done été écrémés. Un pourcentage sensiblement aussi élevé exprime le nombre des laits qui ont été mouillés. Si, avec le Professeur Porcher, on admet, en effet, que la constante moléculaire simplifiée (somme des molécules de lactose évalué en lactose hydraté + somme des molécules de chlorure de sodium évalué isotoniquement en lactose hydraté) ne descend jamais au-dessous de 70 pour les laits normaux et reste, dans la grande majorité des cas, supérieur à 73 , il faut reconnaître que sur les 45 échantillons analysés, 31 étaient certainement mouillés ; ce qui se traduit par un pourcentage voisin de $69 \%$. Si j'ajoute en outre que, dans certains de ces laits, j'ai pu caractériser la présence d'amidon et de silice, j'aurai achevé de faire ressortir fidèlement la physionomie chimique des laits livrés à la consommation lilloise.

$75 \%$ de laits écrémés ;

$69 \%$ de laits mouillés ;

Plusieurs laits adultérés par l'addition d'amidon et de silice.

Tel est le bilan des fraudes qu'a permis d'établir l'analyse d'un certain nombre d'échantillons de cette précieuse denrée alimentaire qu'est le lait.

L'existence d'un délit multiplié autant de fois est évidemment scandaleuse. Elle peut étonner certains esprits aveugles, très facilement enclins à clamer, sans contrôle, l'honnêteté absolue du laitier; elle ne peut cependant surprendre le biochimiste ému depuis longtemps par

(1) Etude de la teneur en matière grasse des laits de la région des Flandres, 1 vol. 110 pages. Imprimerie Centrale du Nord. 
l'indulgence exagérée des tribunaux appelés à juger l'écrémage et le mouillage. Certes, je ne méconnais guère l'importance et l'acuité des controverses soulevées lors de la proclamation de certains verdicts rigoureux rendus à la suite de procès intentés pour fraude du lait.

Je n'ignore pas les faits qui les motivaient; j'ai déjà eu l'occasion en effet, d'exposer publiquement combien la vulgarisation de certaines idées relatives à la variabilité des taux butyreux des laits individuels, risquait d'alimenter ces polémiques et de les multiplier à l'infini.

Je crois cependant avoir montré dans un travail antérieur comment il était possible de clore ces discussions futiles et d'entraver en même temps les manoeuvres frauduleuses des délinquants incorrigibles.

Il appartient, cette fois, au corps médical d'élever la voix pour réclamer des pouvoirs publics l'application de mesures répressives destinées à empêcher que le lait, aliment complet, ne devienne bientôt une simple suspension aqueuse d'amidon ou le véhicule de multiples germes microbiens apportés par l'eau de mouillage.

\section{ANAL YSE DES CASÉINES INDUSTRIELLES (Fin)}

\section{DOSAGE DE L'ACIDITÉ DES CASÉINES INDUSTRIELLES}

\section{par M. DESFLEURS,}

Ingénieur chimiste de la Fabrication française de Produits du Lait.

La correspondance échangée entre MM. BEAU et CHoLLET rappelle la discussion qui eut lieu, il y a quelques années entre MM. W. HoEPFNER et $\mathrm{K}$. JAUDAS d'une part et H. UleX d'autre part.

W. HJ̈PENER et K. JAUDAS (Chemiker Zeitung T. 49, no 38, p. 281, 28 mars 1925) ayant remarqué que dans le dosage de l'acidité des caséines par macération dans l'eau, on obtient des résultats trop forts par suite de la dissolution de matières albuminoïdes qui se combinent à l'alcali, proposèrent de laisser macérer la caséine dans l'alcool à $95 \%$ saturé d'acétate de soude et de titrer une partie aliquote du filtrat.

H. Ulex répondit (Chemiker Zeitung, T. 49, no 92, p. 641-42 du $1^{\mathrm{er}}$ août 1925) que, ni la méthode de LUNGE (agitation de la caséine avec de l'eau et titrage d'une partie du filtrat) ni celle de HöPENER et JAUDAS ne donnent toute l'acidité. On obtient seulement l'acidité superficielle des grains do caséine et non celle qui est à l'intérieur. Aussi proposa-t-il de dissoudre la caséine dans un excès connu de soude titrée et de titrer en retour l'excès d'alcali.

Pour nous, nous croyons que les deux déterminations doivent être conservées.

Comme l'a si bien montré M. le Professeur Poroher, si l'acidité de la caséine chimiquement pure est toujours la même, il n'en est pas de même de l'acidité des caséines industrielles. Pendant la précipitation 